\title{
Music Playlists for People with Dementia: Trialing A Guide for Caregivers
}

\author{
Sandra Garrido ${ }^{\mathrm{a}, \mathrm{b}, *}$, Laura Dunne ${ }^{\mathrm{a}}$, Catherine J. Stevens ${ }^{\mathrm{a}, \mathrm{b}}$ and Esther Chang ${ }^{\mathrm{c}}$ \\ ${ }^{a}$ MARCS Institute for Brain, Behaviour \& Development, Western Sydney University, Penrith, Australia \\ ${ }^{\mathrm{b}}$ School of Social Sciences \& Psychology, Western Sydney University, Penrith, Australia \\ ${ }^{\mathrm{c}}$ School of Nursing \& Midwifery, Western Sydney University, Penrith, Australia
}

Handling Associate Editor: Amy Clements-Cortes

Accepted 5 June 2020

\begin{abstract}
.
Background: Music programs have the potential to provide an effective non-pharmacological tool for caregivers to reduce depression and agitation and increase quality of life in people with dementia. However, where such programs are not facilitated by a trained music therapist, caregivers need greater access to information about how to use music most effectively in response to key challenges to care, and how to pre-empt and manage adverse responses.

Objective: This study reports on the trial of a Guide for use of music with 45 people with dementia and their caregivers in residential care facilities and home-based care.

Methods: The study used a pre-post experimental design in which participants were randomly allocated to a treatment group or a waitlist control group.

Results: Improvements to quality of life were found in the experimental group over the 6-week period. Significant increases in Interest, Responsiveness, Initiation, Involvement, and Enjoyment were reported for individual listening sessions.

Conclusion: The Guide can provide an effective protocol for caregivers to follow in selecting music to manage particular challenges to care, confirming the need for caregivers to be prepared to monitor and manage potential negative responses.
\end{abstract}

Keywords: Aged care, agitation, Alzheimer's disease, arts-based therapies, geriatric depression, health guidelines

\section{INTRODUCTION}

As policy makers and individuals in countries such as Australia and the UK strive to increase independence in older people enabling them to live at home longer [1,2], people who do enter aged care homes are often highly dependent, needing a high level of care [3]. In addition to this challenge, aged care providers face pressures from both market forces and governmental ones to create home-like environments [4] and personalized care models [5] while caring for a great

\footnotetext{
*Correspondence to: Dr. Sandra Garrido, The MARCS Institute for Brain, Behaviour \& Development, Western Sydney University, Locked Bag 1797, Penrith NSW 2751, Australia. Tel.: +61 9772 6585; E-mail: s.garrido@westernsydney.edu.au.
}

diversity of individuals. Thus, aged care facilities are increasingly adopting care programs that can cater to people entering care from a broad range of cultures and lifestyles.

Music programs are gaining in popularity in longterm aged care homes, both due to media interest and for their capacity to provide a personalized, non-pharmacological tool for managing some of the symptoms of dementia such as depression and anxiety. Music can provide a powerful way for individuals with dementia to reconnect with memories, culture, and identity [6]. Music programs implemented by trained music therapists and other non-therapist music interventions can be effective in reducing depression and agitation and increasing quality of life in people with dementia [7]. 
However, results are not universally positive, with multiple studies demonstrating that some people with dementia may not respond to music interventions, or may experience adverse responses [8-12]. Studies reporting mixed results to music interventions with people with dementia highlight the need for music programs to be implemented with care, particularly when working with vulnerable individuals [8]. The potential for adverse responses to music programs are particularly of concern in relation to programs that are not implemented by a trained music therapist, such as where nursing staff, recreational officers, or volunteers may develop programs of music use for residents in aged care homes. Music therapists are trained to anticipate and respond to adverse responses such as overstimulation, emotional flooding, or the triggering of distressing memories [13], while untrained caregivers may have less awareness of the potential for interaction between music and mental health.

In addition, research shows that aged care facilities are often not using music as effectively as they could, with staff knowledge and buy-in a major barrier to the more effective use of music [14]. Aged care staff may tend to view music as a 'babysitter' or mere entertainment, rather than seeing its potential for use as part of treatment and care plans to reduce reliance on pharmacological methods for managing symptoms.

Based on the need for carers of people with dementia to be better equipped with information and procedures for using music with people with dementia, a set of evidence-based guidelines were developed [15]. Music Playlists For People With Dementia: A Guide for Carers, Health Workers and Family (referred to hereafter as 'the Guide') was developed in collaboration with caregivers and aged care providers, and is based on research from the fields of both music psychology and music therapy. The Guide was developed using standard procedures for development of guidelines in healthcare contexts, which included a systematic review of the evidence, and close consultation with caregivers and aged care providers [16, 17]. The purpose of the Guide is to outline strategies for carers to identify individuals who may be vulnerable to negative responses, to offer tactics for the creation of playlists designed to address key challenges to care for individuals with dementia, and to provide suggestions about program monitoring and integration of music into care plans.

This study reports on a trial of the Guide with people with dementia in aged care facilities and home-based care. It was hypothesized that the use of personalized playlists based on implementation of the Guide would result in less decrease in quality of life over time in people with dementia in the treatment group compared to a waitlist control group. It was also hypothesized that use of the playlists would result in increased engagement and attention, and mood improvement during music listening sessions.

\section{METHODS}

\section{Study design}

The study followed a pre-post experimental design in which participants were randomly allocated to a treatment group or a waitlist control group.

\section{Participants}

The original sample included 58 people with dementia (control group $n=23$, treatment group $n=35)$. However, thirteen participants were unable to complete the study due to death $(n=2)$, illness $(n=1)$, change of facility $(n=2)$, or carer circumstances $(n=8)$, leaving a total sample of 45 participants (control group $n=11,6$ males, 5 females; treatment group $n=34,12$ males, 22 females) with a mean age of 81.5 years (SD 9.7). Scores less than 25 on the Standard Mini-Mental State Exam[18] was the inclusion criteria, and participant scores ranged from 25 to 0 with an average score of $9.8(\mathrm{SD}=7.9)$ indicating severe levels of cognitive impairment. The majority had been diagnosed with Alzheimer's disease dementia $(n=10)$, or had a non-specific diagnosis of dementia $(n=23)$, with all others having diagnoses of other types of dementia such as dementia with Lewy bodies, or frontotemporal dementia $(n=12)$. Most participants were living in residential care $(n=36)$, with 8 being cared for at home, and one participant who started the study in home care and then transferred to residential care during the duration of the study.

\section{Procedures}

Aged care facilities and support groups for homebased carers were randomly selected from freely available lists of such organizations in NSW, Australia, and were approached by phone and email. Where an interest in participating was expressed, aged care homes and carer support groups were visited, a presentation given, and information sheets and consent forms distributed. Once institutional support 
was obtained, individuals in aged care homes were referred to the study and dual consent sought from participants with dementia and their guardian (where appropriate). After obtaining written consent, participants, a close family member, or a carer completed the preliminary questionnaire. Inclusion criteria required that participants have scores of 25 or less on the Standardized Mini-Mental State Examination (SMMSE) [18]. However, no participants were excluded at this point (Fig. 1). Participants were then randomly allocated to the treatment group or a waitlist control group. Carers of participants in the treatment group were visited by a member of the research team and given a written copy of the Guide for music use and equipment for playing music. Explanation of how to use the equipment and fill out the study measures was given. Carers were given 2 weeks to prepare music playlists before receiving a phone call from the research team in which any technological issues were addressed and carers were asked to begin using the music playlists. Caregivers used the prepared playlists with the individual with dementia over a 4-6-week period, completing pre-and post measures of each listening session. At the completion of the 4-6-week period, participants completed a post-study questionnaire, and those in the waitlist control group were given a copy of the Guide and music playing equipment for their own personal use.

\section{Measures and materials}

\section{Preliminary questionnaire}

The preliminary questionnaire consisted of demographic questions, the SSMSE [18] to assess cognitive functioning, a baseline outcome measure, and questions that would assist in creation of the playlists. The baseline outcome measure consisted of the Quality of Life in Alzheimer's Disease proxy rating scale (QOL-AD) [19]. The scale consists of 13 aspects of quality of life which are rated on a scale of 1 (Poor) to 4 (Excellent). Reliability in the current study was 0.80 (Cronbach's alpha) at baseline.

Questions to assist in creation of the playlists were as recommended in the Guide. The Vulnerability to Negative Affect Scale - Proxy version (VNADS) (Garrido, Jaroudi, and Moustafa, unpublished results) is a short, 10-item scale developed for use with people with dementia. The VNADS is designed to provide a brief assessment of aspects of mental health that have been identified as increasing vulnerability to negative affective responses to music such as a history of depression, rumination and emotionality. Items

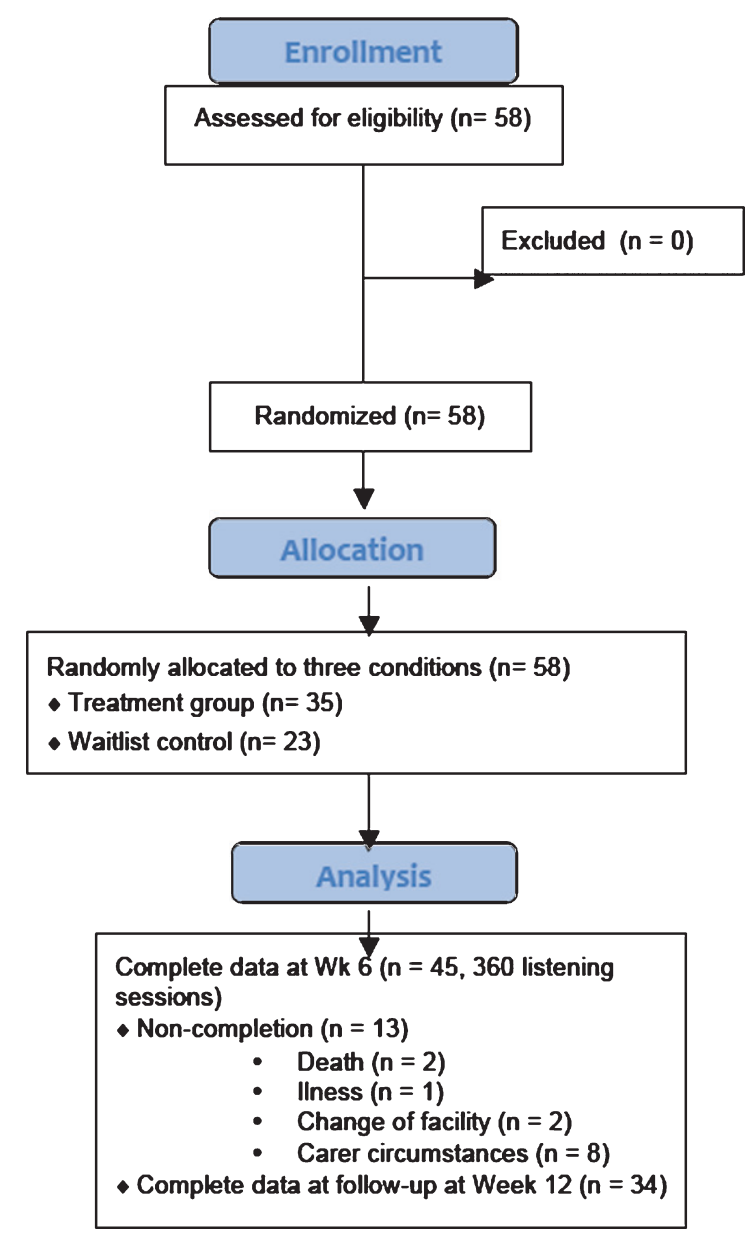

Fig. 1. CONSORT Flow chart of procedure.

were rated on a scale of -2 (Strongly disagree) to 2 (Strongly Agree) with 0 as a neutral score. Reliability testing in the current study returned a Cronbach's alpha of 0.87 . Participants were categorized as No to Low Risk if they scored 1 or under on the scale total (above neutral on 1 or less items on the scale), or Moderate to High Risk if they scored 2 or over on the scale total (above neutral on 2 or more items). Carers were then asked to rate the top three challenges to care experienced by the person with dementia from a list including: 1) agitation or anxiety, 2) withdrawal or apathy, 3) reduced verbal or social engagement, 4) resistance to care situations such as showering, dressing, or eating, 5) restlessness, wandering or falls, and 6) problems sleeping. Finally, music preferences were ascertained based on a questionnaire drawn from Gerdner and colleagues [20]. 


\section{Musical stimuli}

Carers followed the Guide in creating one or more playlists for the person with dementia. As recommended in the Guide, music should be selected based on the individual participant's preferences. However, for people who were classified as Moderate to High Risk according to the VNADS, it was suggested that music should be relatively 'happy' music, i.e., in a major key and containing positive messages, although it was recommended that tempos should be moderate, between 80 and 120 beats per minute (bpm). Other suggestions regarding music tempos were made depending on the particular challenges to care identified by the carer, for example, for individuals with problems sleeping it was suggested that tempos below $80 \mathrm{bpm}$ be selected.

\section{Equipment}

Participants were given a set of Phillips Lightweight Over-Ear Hi-Fi headphones (SHP1900) and a SONY NWZ-B183FMP3 Walkman. They were also given a \$AUD30 iTunes voucher for downloading music. A member of the research team assisted those who needed help to download music from iTunes using the caregivers' personal or office computers.

\section{Measure for pre-post listening sessions}

To assess changes in mood and behavior at each listening session the Music in Dementia Assessment Scale was used (MiDAS) [21]. This scale asks an observer to rate the person with dementia's behavior on 5 aspects by marking a point on a scale of 0 to 100 , and to tick a box if any of 6 behavioral indicators of mood states were present (agitation/aggression, restless/anxious, withdrawn/low mood, relaxed, attentive/interested, cheerful/smiling). This scale was completed prior to and after each listening session by a caregiver or family member.

\section{Post-study survey}

At the end of the 4-6-week period of music use, participants were asked to complete the QOL-AD proxy rating scale again. The same measure was completed at Week 12 of the study as well.

\section{RESULTS}

To test the first hypothesis that quality of life would decrease less over time in the treatment group compared to the control group, a between-within ANOVA was conducted with Group (treatment and control) as the between subject factor, and QoL-AD (Time: baseline and post-study) as the within subject factor. There was no significant main effect of Time $F(1,33)=0.20, p=0.66$. A second analysis with Quality of Life measured at baseline and 12-week follow up was similarly non-significant $F(1,23)=1.66$, $p=0.21$.

Since the QoL-AD assessed a number of factors that were unlikely to be changed by listening to music such as living situation and financial situations, paired sample t-tests for the experimental group and the control group were conducted with individual items from the QoL-AD from baseline and Week 6 measures as the dependent variables (Table 1). Individual items relating to mood, memory, relationships, and life as a whole were selected as items most likely to be influenced by listening to music. Results indicated that after Bonferroni corrections for multiple analyses, 'life as a whole' ratings improved for the experimental group with no change for the control group. Closest relationship also improved in the experimental group but not in the control group ( $p=0.039)$.

To test the second group of hypotheses about the effects of music listening in each session, paired samples t-tests were performed on the five behavioral aspects of the MiDAS. There were 360 listening sessions across 34 participants that were analyzed. All t-tests were significant with Bonferroni corrections for multiple comparisons at the level of $p<0.01$ (see Table 2). Behavioral indicators of Interest, Responsiveness, Initiation, Involvement, and Enjoyment increased significantly from baseline to post listening sessions.

The final items of the MiDAS in which carers were asked to tick a box if evidence of any of the 6 mood states were present were also analyzed for pre-post changes in 313 listening sessions. Participants were categorized as Negative Prior Mood if they had shown evidence of Agitation/Aggression, Restless/Anxious, Withdrawn/Low mood at baseline, or as Positive Prior Mood if they had shown evidence of being Relaxed, Attentive/Interested, or Cheerful/Smiling at baseline. Frequency counts were then made of sessions in which participants mood improved (i.e., shifted to a negative mood of lesser intensity, from a negative to a positive mood, or from a positive mood to a more alert positive mood), mood maintained (i.e., stayed positive or in the same mood state as prior), or deteriorated (i.e., shifted from a positive to a negative mood, or shifted from a negative mood to a negative mood of greater intensity) (Tables 3 and 4). 
Table 1

Descriptive statistics and $p$ values for paired-sample $t$-tests of individual items of QoL-AD

\begin{tabular}{|c|c|c|c|c|c|c|}
\hline \multirow[t]{2}{*}{ Item } & \multicolumn{3}{|c|}{ Experimental group } & \multicolumn{3}{|c|}{ Control group } \\
\hline & $\begin{array}{l}\text { Baseline } \\
\text { mean }(\mathrm{SD})\end{array}$ & $\begin{array}{c}\text { Week } 6 \\
\text { mean }(\mathrm{SD})\end{array}$ & $p$ & $\begin{array}{c}\text { Baseline } \\
\text { mean }(\mathrm{SD})\end{array}$ & $\begin{array}{c}\text { Week } 6 \\
\text { mean }(\mathrm{SD})\end{array}$ & $p$ \\
\hline Mood & $2.02(0.65)$ & $2.26(0.71)$ & 0.083 & $1.57(0.98)$ & $1.86(1.07)$ & 0.356 \\
\hline Family & $2.78(0.93)$ & $3.00(0.78)$ & 0.247 & $2.29(0.76)$ & $2.43(0.98)$ & 0.736 \\
\hline Closest relationship & $2.81(0.96)$ & $3.22(0.80)$ & 0.039 & $2.29(0.76)$ & $2.57(1.13)$ & 0.569 \\
\hline Friends & $1.93(0.83)$ & $2.07(0.83)$ & 0.294 & $1.86(1.22)$ & $1.86(0.90)$ & 0.938 \\
\hline Life as a Whole & $2.00(0.68)$ & $2.30(0.67)$ & $0.009 *$ & $2.0(0.82)$ & $2.0(0.82)$ & 0.901 \\
\hline
\end{tabular}

*Significant at $p<0.01$.

Table 2

Results of paired-samples $t$-tests on behavior as measured by the MiDAS

\begin{tabular}{|c|c|c|c|c|c|c|}
\hline & \multicolumn{2}{|c|}{ Pre-listening } & \multicolumn{2}{|c|}{ Post-listening } & \multirow[b]{2}{*}{$T$} & \multirow[b]{2}{*}{$p$} \\
\hline & $\mathrm{M}$ & SD & $\mathrm{M}$ & $\mathrm{SD}$ & & \\
\hline Interest & 44.75 & 26.59 & 62.60 & 27.15 & 15.521 & $<0.001$ \\
\hline Responsiveness & 47.30 & 26.74 & 64.21 & 28.16 & 12.83 & $<0.001$ \\
\hline Initiation & 42.87 & 28.71 & 56.34 & 31.46 & 12.10 & $<0.001$ \\
\hline Involvement & 44.25 & 27.64 & 61.95 & 28.69 & 12.41 & $<0.001$ \\
\hline Enjoyment & 44.45 & 27.69 & 63.34 & 27.85 & 14.37 & $<0.001$ \\
\hline
\end{tabular}

Table 3

Changes on pre-post mood ratings on 6 behaviors on MiDAS

\begin{tabular}{|c|c|c|c|c|c|c|}
\hline \multirow[t]{2}{*}{ Before } & \multicolumn{6}{|c|}{ After } \\
\hline & $\begin{array}{c}\text { Agitated/ } \\
\text { Aggressive }\end{array}$ & $\begin{array}{l}\text { Restless/ } \\
\text { Anxious }\end{array}$ & $\begin{array}{l}\text { Withdrawn/ } \\
\text { Low mood }\end{array}$ & Relaxed & $\begin{array}{l}\text { Attentive/ } \\
\text { Interested }\end{array}$ & $\begin{array}{c}\text { Cheerful/ } \\
\text { smiling } \\
\end{array}$ \\
\hline Agitated/Aggressive & 0 & 0 & 0 & 11 & 0 & 5 \\
\hline Restless/Anxious & 0 & 9 & 2 & 32 & 11 & 34 \\
\hline Withdrawn/Low mood & 0 & 4 & 5 & 17 & 7 & 16 \\
\hline Relaxed & 0 & 5 & 0 & 21 & 10 & 50 \\
\hline Attentive/Interested & 0 & 1 & 0 & 4 & 4 & 17 \\
\hline Cheerful/Smiling & 0 & 0 & 1 & 2 & 0 & 45 \\
\hline
\end{tabular}

A chi-square test for independence was conducted to examine whether mood outcomes differed significantly based on whether prior mood had been negative or positive. The analysis revealed a significant relationship between prior mood and mood outcome $\chi^{2}(\mathrm{df}=2, \mathrm{~N}=313)=87.90, p<0.001$, $\phi=0.53$. Results indicated that people in a negative mood prior to music listening experienced a mood improvement more frequently than would be expected if the null hypothesis were retained, while people in a positive mood most frequently experienced mood maintenance. Of listening sessions in which participants were demonstrating negative affective states prior to the music listening sessions, mood had improved in 135 cases, was maintained in 14 cases and deteriorated in only 4 cases. Mood deterioration was experienced in only 11 listening sessions overall, with 4 people who had been in a withdrawn mood becoming restless or anxious, 5 people who had been in a relaxed state becoming restless or anxious, one person who had been attentive and
Table 4

$2 \times 3$ Contingency Table of Prior Mood by Mood Changes After Listening

\begin{tabular}{lccc}
\hline $\begin{array}{l}\text { Prior } \\
\text { mood }\end{array}$ & $\begin{array}{c}\text { Mood } \\
\text { improvement }\end{array}$ & $\begin{array}{c}\text { Mood } \\
\text { maintenance }\end{array}$ & $\begin{array}{c}\text { Mood } \\
\text { deterioration }\end{array}$ \\
\hline Negative & $135(95.3)$ & $14(52.3)$ & $4(5.4)$ \\
Positive & $60(99.7)$ & $93(54.7)$ & $7(5.6)$ \\
\hline
\end{tabular}

Expected counts are in brackets.

interested becoming restless or anxious, and one person who had been cheerful and smiling becoming withdrawn or in a low mood (Table 3).

A closer analysis of the 11 listening sessions that had resulted in mood deterioration revealed that this involved 7 participants: 1 who experienced mood deterioration on 4 occasions (out of 16 listening sessions), another who experienced it twice (out of 22 listening sessions), and others who experienced it once (out of 10-16 listening sessions). These participants all had numerous occasions on which listening to music had resulted in an improved 
mood. Three of these individuals had been diagnosed with Alzheimer's disease, two had non-specific diagnoses of dementia, one had early onset dementia and another had a combined diagnosis of Lewy body dementia and Alzheimer's disease. Six of these participants had SMMSE scores below 9 indicating severe cognitive impairment, with only one scoring 16, indicating moderate impairment. Three of the participants, including the participant who experienced mood deterioration on 4 occasions, had been categorized as moderate to high risk on the 10-item VNADS.

\section{DISCUSSION}

The current study aimed to evaluate the effectiveness of a guide to music use for people with dementia which was trialed with patient-caregiver dyads in both long-term residential care and home-based care. Our hypothesis that people with dementia in the treatment group would experience a less steep rate of decline in quality of life than those in the waitlist control group was not supported by the data, although there was some evidence that relationships and 'life as a whole' tended to have improved, at least in the perception of the caregiver.

There are several possible reasons for these results. Firstly, like many studies with people with dementia, this study was challenged by low completion rates, especially in participants in the waitlist control group. Thus, data from the control group likely did not accurately reflect the true decline in quality of life in the original sample. Secondly, it is possible that music is simply not powerful enough to slow long-term decline in the facets of quality of life that were measured in this study. The QOL-AD measures items such as physical health, energy levels, and money issues, facets that are unlikely to be changed by music use. In fact, another common challenge with research relating to people with dementia is finding appropriate measures to capture the key impacts of arts-based interventions. Measures such as the MiDAS or other observational rating scales such as Lawton and colleagues' [22] Observed Emotion Scale provide effective ways to assess the immediate impact of music listening sessions in the short-term. However, measures that can assess the long-term impact of music programs are in short supply. Other indicators such as medicine use, caregiver satisfaction, or frequency of negative behavioral incidents are likely to be more useful for long-term assessment than quality of life measures currently available. Nevertheless, there is a need for more work in this area to develop suitable measures for long-term evaluations. The results of the current study suggest that the impact of music on relationships might be one factor on which future measures could focus.

Despite the lack of positive long-term effects found in the current study, the study revealed that the majority of participants benefited from music listening in multiple ways, supporting our second group of hypotheses. Over 360 listening sessions, participants were observed to have increased levels of interest, responsiveness, involvement, and enjoyment, and were more likely to initiate conversations with caregivers. Out of 313 listening sessions, participants experienced a mood improvement on 195 occasions, experiencing mood deterioration on only 11 occasions. These results indicate that music selected according to the Guide had positive impacts overall. However, the results do highlight that outcomes will not be positive for all individuals on all occasions. As found in previous research, participants with a history of mental illness, patients with Alzheimer's type dementia, or patients with higher rates of cognitive decline are more likely to experience adverse responses [8]. This confirms the need for caregivers to be prepared to monitor listeners and manage potential negative responses, as advised by music therapists [23].

Nevertheless, even participants who experienced mood deterioration on occasion experienced positive effects in most listening sessions. Thus, with careful development and appropriate modification of music use plans it is possible even for high-risk patients to benefit from music programs. As outlined in the Guide, caregivers may need to consider contextual issues such as the equipment used since some people may benefit from the isolating effects of headphones while others may find the use of headphones distressing. Similarly, the listening situation can have an impact on the outcome since some participants may find listening to music an effective way to reduce agitation in response to environmental noise in communal living areas, whereas others may need to be in an isolated place to benefit. Other factors can include the time of day the music is used as well as ensuring that physical needs such as pain management, toileting, and a comfortable temperature are taken care of first. The appropriate conditions and timing of music use needs to be individually determined for each resident and implemented into the patient's overall care plan. 
In addition to the recruitment and measurement issues discussed above, the current study was limited by the fact that it was difficult to assess the degree to which caregivers used and followed the Guide. Staff in residential care facilities as well as homebased caregivers are time-poor and may not have had time to absorb all the material or to implement all the suggestions. Future studies should consider including ways to assess compliance. There is also a need for further research to look at ways to streamline learning materials and processes for effective implementation of music programs for people with dementia. The current study was also limited by a reliance on proxy measures. Studies have shown that proxy measures often do not have high correlations with self-report measures [24]. As noted above, finding effective ways to measure the impact of music programs is an ongoing challenge in this field, given that self-report measures are often not feasible in populations with dementia. Behavioral measurement scales such as the MiDAS which was used in this study can overcome this to some degree by ensuring that ratings are based on observed behavior. However, ideally such ratings should be given by independent observers rather than caregivers as they were in this study. Despite these limitations, the current study adds to the growing body of evidence indicating the benefits of music listening for people with dementia, and can contribute to knowledge about how music programs can best be implemented in care settings.

\section{ACKNOWLEDGMENTS}

This research was supported by a Fellowship to the first author from the National Health and Medical Research Council of Australia.

Authors' disclosures available online (https:// www.j-alz.com/manuscript-disclosures/20-0457r1).

\section{REFERENCES}

[1] Australian Government Department of Health (2019) Home Care Packages Program: Data Report 2nd Quarter 2018-19, Department of Health.

[2] Government Office for Science (2016) Future of an ageing population, Government Office for Science.

[3] Royal Commission into Aged Care Quality and Safety (2019) Medium- and long-term pressures on the system: The changing demographics and dynamics of aged care, Commonwealth of Australia.

[4] Davis S, Byers S, Nay R, Koch S (2009) Guiding design of dementia friendly environments in residential care settings: Considering the living experiences. Dementia 8, 185-203.
[5] Brannelly T, Gilmour JA, O'Reilly H, Leighton M, Woodford A (2017) An ordinary life: People with dementia living in a residential setting. Dementia 18, 757-768.

[6] Evans SC, Garabedian C, Bray J (2017) 'Now he sings'. The My Musical Memories Reminscence Programme: Personalised interaction reminiscence sessions for people living with dementia. Dementia 18, 1181-1198.

[7] Garrido S, Dunne L, Chang E, Perz J, Stevens C, Haertsch M (2017) The use of music playlists for people with dementia: A critical synthesis. J Alzheimers Dis 60, 1129-1142.

[8] Garrido S, Stevens C, Chang E, Dunne L, Perz J (2018) Music and dementia: Individual differences in response to personalized playlists. J Alzheimers Dis 64, 933-941.

[9] Garrido S, Stevens C, Chang E, Dunne L, Perz J (2019) Music and dementia: Musical features and affective responses to personalized playlists. Am J of Alzheimers Dis Other Demen 34, 247-253.

[10] Ziv N, Granot A, Hai S, Dassa A, Haimov I (2007) The effect of background stimulative music on behavior in Alzheimer's patients. J Music Ther 44, 329-343.

[11] Garland K, Beer E, Eppingstall B, O'Connor DW (2007) A comparison of two treatments of agitated behaviour in nursing home residents with dementia: Simulated family presence and preferred music. Am J Ger Psychiat 15, 514521.

[12] Martin PK, Schroeder RW, Smith JM, Jones B (2016) The Roth project - Music and Memory: Surveying the observed benefit of personalized music in individuals with diagnosed or suspected dementia. Alzheimers Dement 12, P988.

[13] Moore KS, Five problems music can create, https://www. psychologytoday.com/us/blog/your-musical-self/201408/ 5-problems-music-can-create, Accessed 28 Nov 2019.

[14] Garrido S, Dunne L, Perz J, Chang E, Stevens C (2018) The use of music in aged care facilities: A mixed methods study. J Health Psychol 15, 765-776.

[15] Garrido S, Dunne L, Stevens C, Chang E, Perz J (2019) Music playlists for people with dementia: A guide for carers, health workers and family, Western Sydney University.

[16] White M (2010) Developing guidelines for good practice in participatory arts-in-health-care contexts. J Appl Arts Health 1, 139-155.

[17] Council of Medical Specialty Societies (2017) CMSS principles for the development of specialty society clinical guidelines. Council of Medical Specialty Societies, Chicago, IL. https://cmss.org/wp-content/uploads/2017/11/ Revised-CMSS-Principles-for-Clinical-Practice-GuidelineDevelopment.pdf

[18] Vertesi A, Lever JA, Molloy DW, Sanderson B, Tuttle I, Pokoradi L, Principi E (2001) Standardized Mini-Mental State Examination: Use and interpretation. Can Fam Physician 47, 2018-2023.

[19] Logsdon RG, Gibbons LE, McCurry SM, Teri L (1999) Quality of life in Alzheimer's Disease: Patient and caregiver reports. J Ment Health Aging 5, 21-32.

[20] Gerdner LA, Hartsock J, Buckwalter KC (2000) Assessment of Personal Music Preference (Patient Version). University of Iowa, Iowa. http://www.carepartnermentoring.com/ music/Music\%20Preference\%20Questionnaire\%20Patient $\% 20$ Version.pdf

[21] McDermott O, Orrell M, Ridder HMO (2015) The development of Music in Dementia Assessment Scales (MiDAS). Nord J Music Ther 24, 232-251.

[22] Lawton MP, Van Haitsma K, Klapper JA (1999) Observed affect and quality of life in dementia: Further affirmations and problems. J Ment Health Aging 5, 69-81. 
[23] Clements-Cortes A, Pearson C, Chang K (2015) Creating effective music listening opportunities. Baycrest, Toronto, Ontario. http://www.baycrest.org/care/culturearts-innovation/therapeutic-arts/music-therapy/creatingeffective-music-listeningopportunities
[24] Moyle W, Murfield JE, Griffiths SG, Venturato L (2012) Assessing quality of life of older people with dementia: A comparison of quantitative self-report and proxy accounts. J Adv Nurs 68, 2237-2246. 